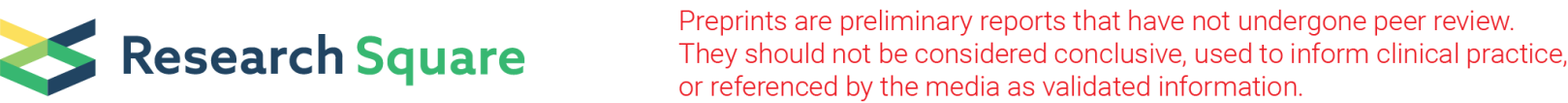

\section{Cerebral Stroke Lesion Location and the Risk of Post-Stroke Pain A Systematic Review and Meta- Analysis}

\section{Sheng Yuan}

Chinese Academy of Medical Sciences and Peking Union Medical College Jiaxin Wang

Huazhong University of Science and Technology Tongji Medical College Jia Yu

The ministry of Science and Technology

\section{Xiaojiao Wang}

The Ministry of Science and Technology

\section{Qiang Li}

The Ministry of Science and Technology

\section{Yufen Zhang}

Huazhong University of Science and Technology Tongji Medical College

\section{Sehui Ma}

Huazhong University of Science and Technology Tongji Medical College

\section{Yao Yi}

Huazhong University of Science and Technology Tongji Medical College Hongyan Yu

Huazhong University of Science and Technology Tongji Medical College

\section{Dian Yu}

Huazhong University of Science and Technology Tongji Medical College

\section{Fei Lv}

Wuhan University Renmin Hospital

\section{Shaojun Li}

Wuhan First Hospital

\section{Dan Feng}

Wuhan First Hospital

\section{Lei Pei ( $\nabla$ leipei@wustl.edu )}

Washington University in Saint Louis School of Medicine 
Keywords: Cerebral Stroke, Post-stroke pain, Lesion location, Meta-analysis

Posted Date: January 8th, 2020

DOI: https://doi.org/10.21203/rs.2.20367/v1

License: (c) (1) This work is licensed under a Creative Commons Attribution 4.0 International License. Read Full License 


\section{Abstract}

\section{Background}

Post-stroke pain (PSP) is generally a severe complication in stroke rehabilitation. It is always associated with allodynia and hyperalgesia to nociceptive stimuli. Previous studies believed that PSP was related with tissue injury at certain brain territory, possibly thalamic lesion. However, there is very little evidence that the risk of PSP has a salient association with the side of lesion location (left or right hemisphere). The aim of this study was to assess the relationship between the occurrence of PSP and the lesion location of stroke.

Methods

We selected relevant literatures by searching EMBASE, ISI Web of Science, PubMed, and systematically reviewed available publications according to predetermined inclusion criterion. Subgroup analyses were performed according to the subtype of PSP. The pooled risk estimates were calculated by using a randomized-effects model.

Results

A total of 19 studies involving 2038 patients suffered from cerebral stroke were included. Meta-analysis showed no significant relationship between brain lesion location and the risk of PSP (OR=0.95 95\% Cl 0.74-1.23). Subgroups analyses showed that the incidence of post-stroke shoulder pain (PSSP) or central post-stroke pain (CPSP) was also not associated with the side of brain lesion (PSSP: OR=0.91, 95\% $\mathrm{Cl}$ 0.57-1.46; CPSP: OR=0.99, 95\% $\mathrm{Cl} 0.70-1.40$ ). The pooled result of prospective studies had similar result ( $R R=1.02,95 \% \mathrm{Cl} 0.84-1.25)$. Evidence of publication bias has not been found using visual inspection of a funnel plot and Begg's and Egger's tests.

\section{Conclusions}

Our systematic meta-analysis suggests that the lesion location of stroke has no significant association with the incidence of post-stroke pain. Further studies are needed to detect the risk factors for PSP, which may contribute to the prevention, identification and management of PSP.

\section{Background}

Stroke is the leading cause of morbidity and mortality worldwide, and pain is one of the serious common complications after stroke, which brings a heavy burden to the patients and exerts negative effects on the rehabilitation of stroke [1]. Post-stroke pain (PSP) presents with a variety of pain, including neuropathic pain, namely central post-stroke pain (CPSP), nociceptive pain (musculoskeletal pain, shoulder pain, spasticity-related pain), headache and so on [2]. The estimated prevalence rate of PSP ranges from 1045.8\% [3], suggesting a difficulty in reliably, accurately, and consistently identifying PSP. 
It is known that the incidence of PSP is associated with brain lesion after stroke [4]. For example, previous studies have shown that the occurrence of PSP may be caused by the lesion of the dorsolateral prefrontal cortex, rostral anterior cingulate cortex, amygdala, hippocampus, periaqueductal gray, rostral ventromedial medulla, and medial thalamus [5]. These studies are mainly based on retrospective data without using clinical examination or widely agreed criteria for distinguishing different types of pain [6]. Whether the lesion location of stroke is related with the risk of post-stroke pain remains unknown.

In this systematic meta-analysis, PSP patients were enrolled underwent bedside clinical examination. And using widely accepted criteria, they were diagnosed with different types of pain after stroke. We aimed to seek reliable information in the literature, present the relationship between the lesion location of stroke and the risk of PSP and hope to provide evidences for the diagnosis and prevention of PSP.

\section{Methods}

This work was conducted in accordance with the Preferred Reporting Items for Systemic Reviews and Meta-analysis (PRISMA) guidelines (http://www.prisma-statement.org/). All studies that included an assessment of pain in patients who suffered from stroke and clear data to relate the presence of pain to the site of the stroke lesion were eligible for inclusion. In addition, our study has been registered in the International Prospective Register of Systematic Reviews (PROSPERO) (registration number: CRD42019139928).

\section{Search strategy}

A systematic literature search was conducted by searching the online databases EMBASE, ISI Web of Science and PubMed. We used the terms "pain" in combination with "stroke". The complete retrieval strategy for PubMed is: (stroke[MeSH Terms]) OR stroke[Title/Abstract]) OR post-stroke[Title/Abstract]) OR poststroke[Title/Abstract]) OR post stroke[Title/Abstract]) OR Strokes[Title/Abstract]) OR Cerebrovascular Accident[Title/Abstract]) OR Cerebrovascular Accidents[Title/Abstract]) OR Apoplexy[Title/Abstract]) OR Vascular Accident, Brain[Title/Abstract]) OR Brain Vascular Accident[Title/Abstract]) OR Brain Vascular Accidents[Title/Abstract]) OR Vascular Accidents, Brain[Title/Abstract])) AND (pain[MeSH Terms]) OR pain[Title/Abstract]) OR pains[Title/Abstract]) OR ache[Title/Abstract]) OR aches[Title/Abstract]) OR headache[Title/Abstract]) OR headaches[Title/Abstract]). All studies were updated to 31st December, 2019.

In addition, the reference lists of the included studies and relative reviews or reports were also incorporated into the search boundary. No filter for methodology of the studies was applied to pledge the comprehensiveness of the search strategy. After removed the duplicated records, titles and abstracts of all studies were screened in the first round. More research details could be obtained by scrutinizing the full text. All articles were screened independently by two investigators (SY and JW). Their results reached consensus. If their opinions were divided, agreement will be reached with the third researcher (YZ). 


\section{Study selection}

We included studies and data which met the following criteria: (1) the studies must have detected the association between lesion site of stroke and post-stroke pain; (2) the Odds Ratio (OR) and the corresponding 95\% confidence interval (Cl) could be calculated from the data provided; (3) CT or MR scanning was used to confirm the lesion location of stroke; (4) the search was restricted to studies on human subjects and the findings were published in English. Among duplicate studies, we selected the result with largest number of original data.

\section{Data extraction}

Two independent investigators (SY and JW) selected the studies strictly according to the inclusion criteria. Disagreements in data extraction between the two investigators were resolved through group discussions with the third investigator (YZ). The data we collected and extracted from every selected study included source of patient, research type, sample size, number of PSP, PSP identification and assessment, types of pain, types of stroke, lesion location, history of stroke and pain. We had not contacted the corresponding author to collect any required unpublished information.

\section{Quality assessment of selected studies}

The quality of included cohort studies and case-control studies was assessed by the Newcastle-Ottawa scale (NOS) [7]. The NOS uses a 'star' rating system to evaluate quality based on the three following aspects: selection, comparability, and exposure (case-control studies) or outcome (cohort studies). Scores were ranged from 0 (worst) to 9 stars (best). The scores from 0 to 3 were considered as lower study quality, scores from 4 to 5 were considered as intermediate study quality, and scores from 6 to 9 were considered as high quality. Study with a score of no less than 4 was included. Furthermore, the quality of included cross-sectional studies was assessed by an 11-item questionnaire which was recommended by Agency for Healthcare Research and Quality (AHRQ) (http://www.ncbi.nlm.nih.gov/books/NBK35156). The issues were answered by 'YES' (for 1 point), 'NO' (for 0 ) or 'UNCLEAR' (for 0 ). The quality of the articles was divided into the three categories according to the following criteria: low quality $=0-3$ points; intermediate quality $=4-7$ points; high quality $=8-11$ points. Study with low quality will be excluded. Each study was rated independently by another two investigators (SL and DF).

\section{Statistical analysis}

To estimate the heterogeneity of the results, the analysis was performed in two stages. Firstly, all studies were included to calculate and analyze the relationship of the lesion location of stroke and PSP; secondly, we performed subgroups analyses based on the different subtype of the PSP: central post-stroke pain (CPSP) and post-stroke shoulder pain (PSSP). In addition, high-quality prospective cohort studies were 
considered to have a high level of evidence. Therefore, we divided the prospective cohort study into a subgroup to assess the association between stroke and PSP. Stata software (version 12.0; College Station, TX, USA) were used for statistical analysis and to obtain forest plots for presenting the results of pooled analysis and individual studies. $\mathrm{I}^{2}$ statistic were used to examine the heterogeneity among studies. A random-effects model was used to estimate the pooled ORs and $95 \% \mathrm{Cls}$. Sensitivity analysis was applied to evaluate the stability of the results. During the analysis each study was removed at a time and the rest were used to recalculate the pooled OR to find whether the results were affected statistically significant. The Begg's funnel plot and Egger's test were used to evaluate publication bias [8]. Two-sides $P$ values less than 0.05 were considered as significant.

\section{Results}

\section{Characteristics of the included studies}

The initial search included 10439 citations. After screening through title and abstracts, 9828 citations were excluded initially based on the criteria outlined above. Then, after screening based on the content of full text, 272 citations were included for further assessment. Finally, 19 original reports with 2038 stroke patients were selected and judged eligible for inclusion in the meta-analysis after screening through inclusion/exclusion criteria. The selection process of the studies and specific reasons for exclusion are displayed in Fig. 1. The clinic features and demographics of the studies are outlined in Additional file 1 (Table S1).

\section{Association between lesion location of stroke and the incidence of PSP}

Firstly, we pooled all the studies together to investigate the association between lesion location of stroke and incidence of PSP. The 19 studies involved 2038 subjects including 967 patients with left hemispheric stroke and 1071 patients with right hemispheric stroke. The pooled OR with $95 \% \mathrm{Cl}$ for the association of stroke location and PSP risk was $0.95(0.74-1.23)\left(I^{2}=12.4 \%\right)($ Fig. 2). This result suggests no significant differences in risk of PSP in relation to the lesion location of stroke.

\section{Subgroup analyses}

Next, we did the subgroup analyses of the two different types of PSP, mainly the post-stroke shoulder pain (PSSP) and the central post-stroke pain (CPSP) (Fig. 3) from the above 19 studies. Because musculoskeletal pains, spasticity-related pain and headache were not appeared in the included 19 reports, the analyses on these types of pain could not conduct. The results showed no significant association between lesion location and the incidence of PSSP or CPSP. We also did subgroup analyses 
by pooling the prospective studies together, but no positive result was found $(\mathrm{RR}=1.02,95 \% \mathrm{Cl} 0.84-$ 1.25) (Fig. 4).

\section{Quality assessment and sensitivity analyses}

Quality assessment of the studies was shown in Additional file 2 (Table S2) and Additional file 3 (Table S3). All the 19 included studies scored no less than 4 based on the NOS and AHRQ. We then conducted sensitive analyses to assess the influence of each study on the pooled OR. As shown in Fig. 5, when any single study was removed, the corresponding ORs were not significantly changed, suggesting high stability of this meta-analysis.

\section{Publication bias}

Begg's funnel plot and Egger's linear regression were performed to assess the publication bias of the included studies. The shapes of the funnel plots did not reveal any evidence of obvious asymmetry. The results of Egger's test also showed that there were not statistically significant differences. (total: $P_{\text {Egger's }}$ test $=0.732$; prospective studies: $P_{\text {Egger's test }}=0.745$ )

\section{Risk of bias assessment for included studies}

\section{Bias from study design}

Eight included studies are prospective studies $[9,10,12,13,15,19,20,22,23]$, they collected baseline information of stroke patients and followed at periodic intervals to examine the incidence of PSP (Fig. 6). Other studies consist of retrospective studies and cross-sectional studies, which are less adept at proving a causal relationship and were difficult to confirm the explicit time of PSP occurrence.

\section{Bias from inclusion criterion}

Nine studies only included patients with first-ever stroke [13-15, 18-24], while 2 studies did not exclude patients with previous studies $[9,10]$. Eight studies did not mention the selection criterion about stroke history $[11,12,16,17,24-27]$. Since different lesion site of stroke may have different effect, it is difficult to distinguish the impact of recurrent stroke with different lesion location. Similarly, three studies have not excluded patients with history of pain prior to stroke and 5 studies have not given the exclusion criterion about history of pain, which may interfere with the diagnosis of PSP (Fig. 7).

\section{Bias from subtype of stroke}


Most of the included studies have involved both ischemic and hemorrhagic stroke. 4 studies only included patients with ischemic stroke [13, 19, 22, 23], while 1 study [15] discussed CPSP only about hemorrhagic stroke and 1 study [18] has not given the specific stroke type. Because of the different mechanism of pathogenesis, it is better to describe the impact of hemorrhagic and ischemic stroke respectively.

\section{Bias from PSP definition}

There are several methods for pain measurement, such as Visual Analogue Scale (VAS) ( 5 studies $[9,17$, $18,20,24])$, numerical rating system (NRS) (5 studies [14, 15, 22, 23, 25]), Douleur neuropatathique en 4 questions (DN4) (3 studies [22, 25, 27]), The Leeds Assessment of Neuropathic Symptoms and Signs (LANSS) (2 studies [24, 25]) or combined assessment. The check of PSSP is always companied with assessment of upper-limp. Moreover, there is also part of overlap between PSSP and CPSP, which adds to the complexity of diagnosis (Fig. 8) [2].

\section{Discussion}

Post-stroke pain restricts patients' daily life after stroke and needs close attention in diagnosis and poststroke care [28]. It is of great significance to explore the potential risk factors of PSP for clinical diagnosis and treatment as a consequence. Many factors have been proved to be associated with the incidence of PSP. Significant risk factors for PSP included female gender, greater alcohol intake, increased severity of stroke, depression, diabetes and vascular disease of blood vessels supplying the lower limbs [29]. Other characteristics associated with the incidence of PSP include hemorrhagic stroke and initial mobility limitation [30]. In addition, older people are more likely to develop PSP from 3 to 18 months after stroke onset [27]. Even though, there was no direct evidence to support the hypothesis that the lesion location of stroke was an important confounder for the diagnosis of PSP. It is reported that the right hemisphere was more frequently relative with pain [31]. But further studies with larger sample size of patients are needed to confirm this conclusion.

Our present study, for the first time, systematically reveals that the lesion side of stroke (left or right hemisphere) has no relationship with the incidence of post-stroke pain, which means the morbidity of post-stroke pain is similar in both left hemisphere stroke and right hemisphere stroke. Our study has also first analyzed two types of PSP, central post-stroke pain (CPSP) and post-stroke shoulder pain (PSSP), respectively. Our results show that neither the risk of CPSP nor the risk of PSSP is significantly associated with the site of stroke induced brain injury. In addition, the subgroup analysis of prospective cohorts proved that lateralization of stroke has no significant association with PSP.

Although this meta-analysis proved that the lesion location of stroke was not associated with the risk of PSP, the correlation between the lesion site and the incidence of PSP cannot be completely ruled out. Identification of PSP has always been a big challenge for physicians due to the specific natural history and various factors. For example, post-stroke patients may have motor problems, impairment of 
language skills, cognitive decline or depressive tendencies, which may lead to the patients' neglect or inattention to pain [32,33]. Furthermore, pain is a subjective sensuousness. Despite many scales, such as VAS, DN4 and LANSS, were used for pain assessment in clinical practice, there was no specific scale for PSP assessment so far, which may cause heterogeneity in pain assessment between the included studies. In addition, only CPSP and PSSP were included in subgroup analysis. Other common subtype of PSP, such as pain secondary to spasticity, complex regional pain syndrome, headache or any combinations of at least two typical syndromes, were not analyzed due to fewer cases. ${ }^{1}$ Not only was the subgroup analysis of PSP not complete, but also the classification of pain should be considered. Constant pain, spontaneous intermittent pain or hyperalgesia/allodynia may correspond to disparate region of injury. ${ }^{8}$ Besides, the time interval between stroke and pain assessment varied widely from several weeks to years in the included studies, which made it difficult to define the time of illness.

\section{Strengths And Limitations}

Our study clearly points out that lesion location (left or right hemisphere) has no significant relationship with the incidence of PSP. As shown in the subgroup analysis and sensitivity analysis, the result is stable and has low heterogeneity. However, we must admit that there are several limitations in our study. Firstly, the possibility of information and selection biases and unidentified confounders cannot be completely excluded because all the included studies were observational. Secondly, the analysis of the lesion site after stroke is not very comprehensive. In fact, anatomical location such as thalamus or spinal cord may also be related with PSP [2]. Thirdly, the time interval between stroke and pain assessment varied widely from several weeks to years. Cumulative effect contributes to the high incidence of PSP for long time interval studies, which may have influence on our results. Lastly, we restricted our search strategy to articles published in English. While, articles with potentially high-quality data that were published in other languages were not included because of anticipated difficulties in obtaining accurate medical translation.

\section{Conclusions}

Overall, our review, which used the highest protocol for systematic reviews of the relevant literature indicates that there is no significant relationship between the lesion location and the risk of post-stroke pain. Further investigation of PSP with brain imaging and other advanced methodologies may give way to the development of clinical trials that can address treatment options for PSP. In addition, more highquality prospective studies with homogeneity should be conducted to demonstrate the correlation of PSP and the location of stroke. Innovation the assessment and identifying the risk of PSP may contribute to individualization of PSP prediction and help improving patients' quality of life.

\section{Abbreviations}

AHRQ:Agency for Healthcare Research and Quality; CPSP:central post-stroke pain; DN4:Douleur Neuropatathique en 4 questions; LANSS:Leeds Assessment of Neuropathic Symptoms and Signs; NOS:Newcastle-Ottawa Scale; NRS:Numerical Rating System; ORs:odd ratio; PRISMA:Preferred Reporting 
Items for Systemic Reviews and Meta-analysis; PSP:post-stroke pain; PSSP:post-stroke shoulder pain; RRs:relative risk; VAS:Visual Analogue Scale

\section{Declarations}

\section{Acknowledgements}

We would like to thank Prof. Jeremiah Morrissey from Department of Anesthesia, Washington University in Saint Louis for his valuable and constructive suggestions on study design and article writing.

\section{Funding}

This work was supported by National Natural Science Foundation of China (Grant number 81571078ه 81870932 to L. Pei) and Research Project of Health and Family Planning Commission of Wuhan Municipality (WX17D04) to D. Feng.

\section{Availability of data and materials}

All data and articles supporting the conclusions of this systematic review are included within the systematic review.

\section{Author Contributions}

LP designed the study. SY and JW conducted the analysis, interpreted the data and drafted the manuscript. JY contributed to the literature investigation. XW contributed to the supervision of this study. QL conducted the software validation and application. SL, DF, YZ, SM, YY, HY, DY and FL contributed to the interpretation of the data and critical revision of the manuscript for important intellectual content. All authors read and approved the final manuscript.

\section{Ethics approval and consent to participate}

Not applicable

\section{Consent for publication}

Not applicable

\section{Competing interests}

The authors declare that they have no competing interests.

\section{References}

1. Harrison RA, Field TS. Post stroke pain: identification, assessment, and therapy. Cerebrovasc Dis 2015;39:190-201. 
2. Klit H, Finnerup NB, Jensen TS. Central post-stroke pain: clinical characteristics, pathophysiology, and management. Lancet Neurol. 2009;8:857-68.

3. Paolucci S, losa M, Toni D, Barbanti P, Bovi P, Cavallini A, et al. Prevalence and Time Course of PostStroke Pain: A Multicenter Prospective Hospital-Based Study. Pain Med 2016;17:924-30.

4. Kim JS. Post-stroke pain. Expert Rev Neurother 2009;9:711-21.

5. Seifert CL, Mallar Chakravarty M, Sprenger T. The complexities of pain after stroke--a review with a focus on central post-stroke pain. Panminerva Med 2013;55:1-10.

6. Vaegter HB, Andersen PG, Madsen MF, Handberg G, Enggaard TP. Prevalence of neuropathic pain according to the IASP grading system in patients with chronic non-malignant pain. Pain Med 2014;15:120-7.

7. Stang A. Critical evaluation of the Newcastle-Ottawa scale for the assessment of the quality of nonrandomized studies in meta-analyses. Eur J Epidemiol. 2010;25:603-5.

8. Egger M, Davey Smith G, Schneider M, Minder C. Bias in meta-analysis detected by a simple, graphical test. BMJ 1997;315:629-34.

9. Adey-Wakeling Z, Arima H, Crotty M, Leyden J, Kleinig T, Anderson CS et al. Incidence and associations of hemiplegic shoulder pain poststroke: prospective population-based study. Arch Phys Med Rehabil 2015;96:241-47.e1.

10. Andersen G, Vestergaard K, Ingeman-Nielsen M, Jensen TS. Incidence of central post-stroke pain. Pain 1995;61:187-93.

11. Blennerhassett, JM, Gyngell K, and Crean R. Reduced active control and passive range at the shoulder increase risk of shoulder pain during inpatient rehabilitation post-stroke: an observational study. J Physiother 2010;56:195-9.

12. Bugnicourt JM, Garcia PY, Canaple S, Lamy C, Godefroy O. Central neuropathic pain after cerebral venous thrombosis is not so uncommon: an observational study. J Neurol 2011;258:1150-6.

13. Harno H, Haapaniemi E, Putaala J, Haanpää M, Mäkelä JP, Kalso E et al. Central poststroke pain in young ischemic stroke survivors in the Helsinki Young Stroke Registry. Neurology 2014;83:1147-54.

14. Kim NY, Lee SC, An YS, Shin JC, Kim YW. Metabolic Changes in Central Poststroke Pain Following Thalamic Intracerebral Hemorrhage: An 18F-FDG PET Study. Clin Nucl Med 2018;43:e62-e66.

15. Kim YH, Jung SJ, Yang EJ, Paik NJ. Clinical and sonographic risk factors for hemiplegic shoulder pain: A longitudinal observational study. J Rehabil Med 2014;46: 81-7.

16. Krause T, Asseyer S, Geisler F, Fiebach JB, Oeltjenbruns J, Kopf A. Chronic sensory stroke with and without central pain is associated with bilaterally distributed sensory abnormalities as detected by quantitative sensory testing. Pain 2016;157:194-202.

17. Lindgren I, Brogardh C. Poststroke shoulder pain and its association with upper extremity sensorimotor function, daily hand activities, perceived participation, and life satisfaction. PM $R$ 2014;6:781-9. 
18. Niessen M, Janssen T, Meskers C, Koppe P, Konijnenbelt M, Veeger D. Kinematics of the contralateral and ipsilateral shoulder: a possible relationship with post-stroke shoulder pain. J Rehabil Med 2008;40:482-6.

19. Osama A, Abo Hagar A, Elkholy S, Negm M, Abd El-Razek R. Orabi M Central post-stroke pain: predictors and relationship with magnetic resonance imaging and somatosensory evoked potentials. Egypt J Neurol Psychiatr Neurosurg 2018;54:40.

20. Pompa A, Clemenzi A, Troisi E, Di Mario M, Tonini A, Pace L et al. Enhanced-MRI and ultrasound evaluation of painful shoulder in patients after stroke: a pilot study. Eur Neurol 2011;66:175-81.

21. Rajaratnam BS, Venketasubramanian N, Kumar PV, Goh JC, Chan YH. Predictability of simple clinical tests to identify shoulder pain after stroke. Arch Phys Med Rehabil 2007;88:1016-21.

22. Roosink M, Renzenbrink GJ, Buitenweg JR, Van Dongen RT, Geurts AC, IJzerman MJ. Persistent shoulder pain in the first 6 months after stroke: results of a prospective cohort study. Arch Phys Med Rehabil 2011;92:1139-45.

23. Roosink M, Renzenbrink GJ, Buitenweg JR, van Dongen RT, Geurts AC, ljzerman MJ. Somatosensory symptoms and signs and conditioned pain modulation in chronic post-stroke shoulder pain. J Pain 2011;12:476-85.

24. Şahin-Onat Ş, Ünsal-Delialioğlu S, Kulaklı F, Özel S. The effects of central post-stroke pain on quality of life and depression in patients with stroke. J Phys Ther Sci 2016; 28:96-101.

25. Vukojevic Z, Dominovic Kovacevic A, Peric S, Grgic S, Bjelica B, Basta I et al. Frequency and features of the central poststroke pain. J Neurol Sci 2018;391:100-3.

26. Weimar C, Kloke M, Schlott M, Katsarava Z, Diener HC. Central poststroke pain in a consecutive cohort of stroke patients. Cerebrovasc Dis 2002;14:261-3.

27. Zeilig G, Rivel M, Weingarden H, Gaidoukov E, Defrin R. Hemiplegic shoulder pain: evidence of a neuropathic origin. Pain 2013;154:263-71.

28. Hansen AP, Marcussen NS, Klit H, Andersen G, Finnerup NB, Jensen TS Pain following stroke: a prospective study. Eur J Pain 2012;16:1128-36.

29. Wissel J, Manack A, Brainin M. Toward an epidemiology of poststroke spasticity. Neurology 2013;80 Suppl 2:13-9.

30. Klit H, Finnerup NB, Andersen G, Jensen TS. Central poststroke pain: a population-based study. Pain 2011;152:818-24.

31. Merskey H, Watson GD. The lateralisation of pain. Pain 1979;7:271-80.

32. Baron R, Binder A, Wasner G. Neuropathic pain: diagnosis, pathophysiological mechanisms, and treatment. Lancet Neurol 2010;9:807-19.

33. Hope TMH, Leff AP, Prejawa S, Bruce R, Haigh Z, Lim L et al. Right hemisphere structural adaptation and changing language skills years after left hemisphere stroke. Brain 2017;140:1718-28.

\section{Figures}



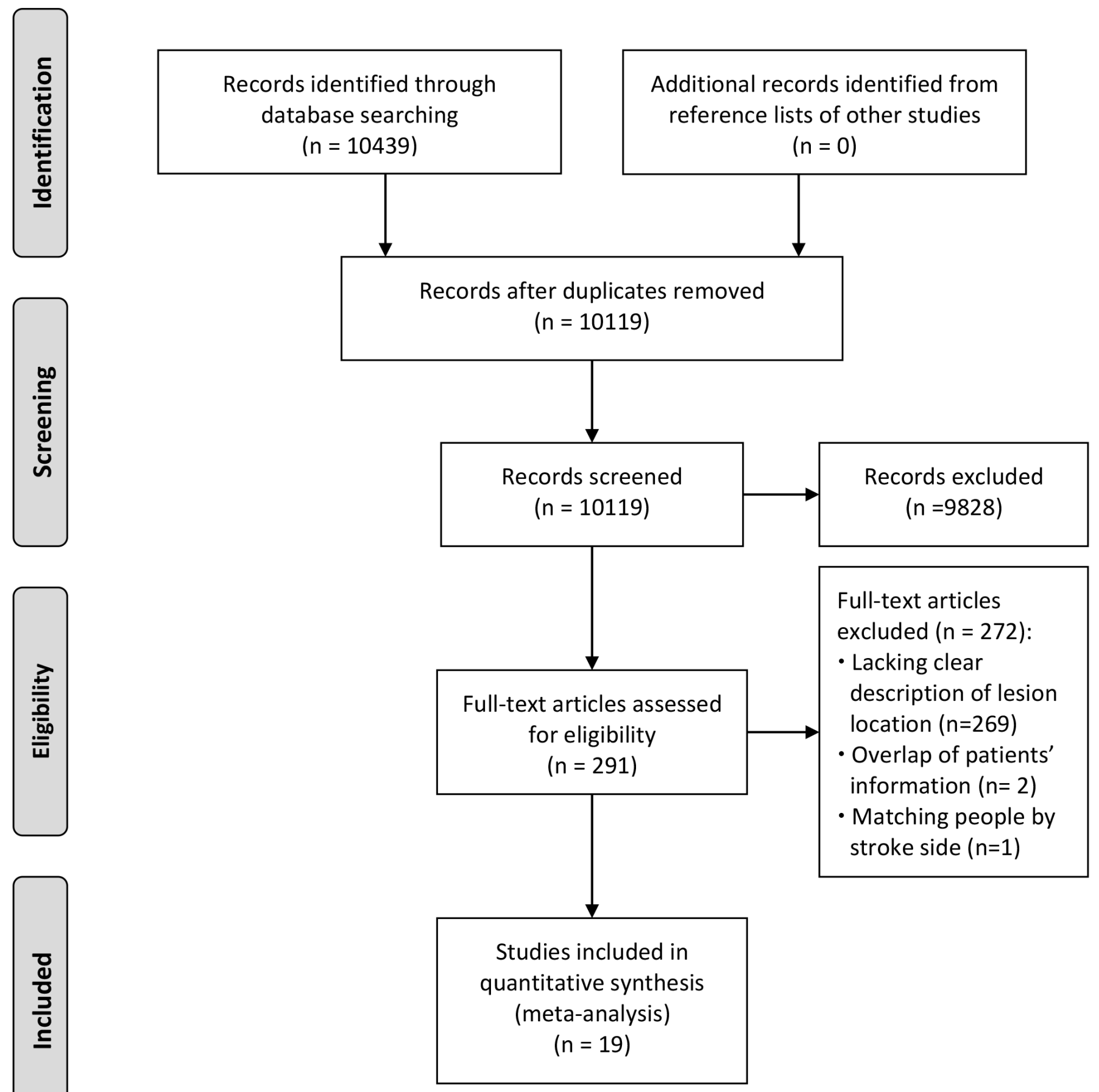

\section{Figure 2}

Flow chart of the selection of studies and specific reasons for the exclusion from the present metaanalysis. 
Study

$\%$

ID

OR $(95 \% \mathrm{CI}) \quad$ Weight

Adey-Wakeling, Z. (2015)

Andersen, G. (1995)

Blennerhassett, J. M. (2010)

Bugnicourt, J. M. (2011)

Harno, H. (2014)

Kim, N. Y. (2018)

Kim, Y. H. (2014)

Krause, T. (2016)

Lindgren, I. (2014)

Niessen, M. (2008)

Osama, A. (2018)

Pompa, A. (2011)

Rajaratnam, B. S. (2007)

Roosink, M.(1) (2011)

Roosink, M.(2) (2011)

Sahin-Onat, S. (2016)

Vukojevic, Z. (2018)

Weimar, C. (2002)

Zeilig, G. (2013)

Overall (I-squared $=12.4 \%, \mathrm{p}=0.302$ )

NOTE: Weights are from random effects analysis

$\begin{array}{ccc}1 & 1 & 1 \\ 0.05 & 1.00 & 20.00\end{array}$

Figure 4

The forest plot of odd ratio (ORs) with $95 \% \mathrm{Cl}$ for the overall association of stroke lesion location with PSP risk. 
Study

\section{ID}

$\%$

OR $(95 \% \mathrm{CI}) \quad$ Weight

\section{Figure 6}

The forest plots of odd ratio (ORs) with 95\% Cl for the association of stroke location with PSSP/CPSP risk. 


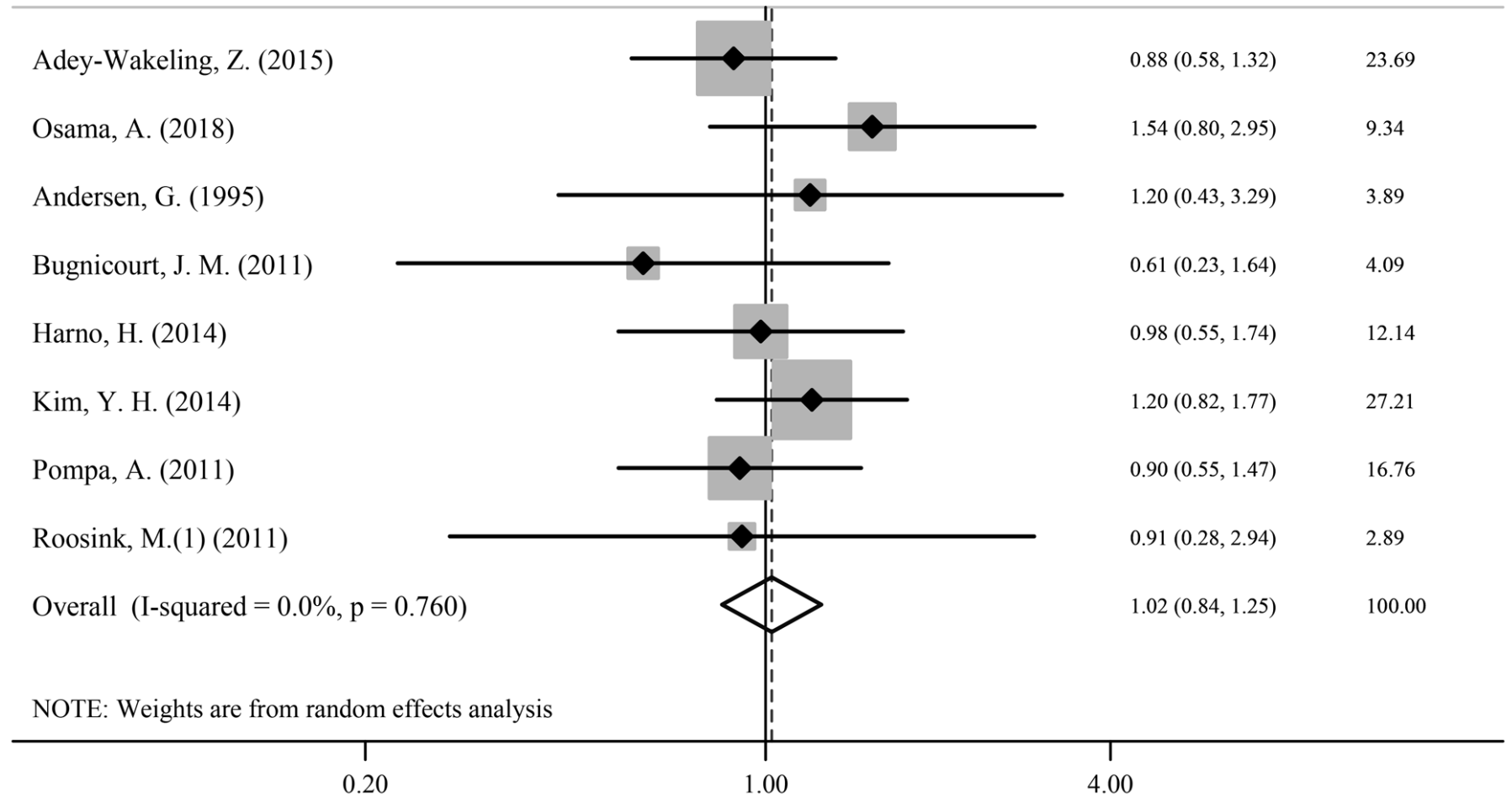

\section{Figure 8}

The forest plots of relative risk (RRs) with $95 \% \mathrm{Cl}$ for the association of stroke location with PSP risk. 


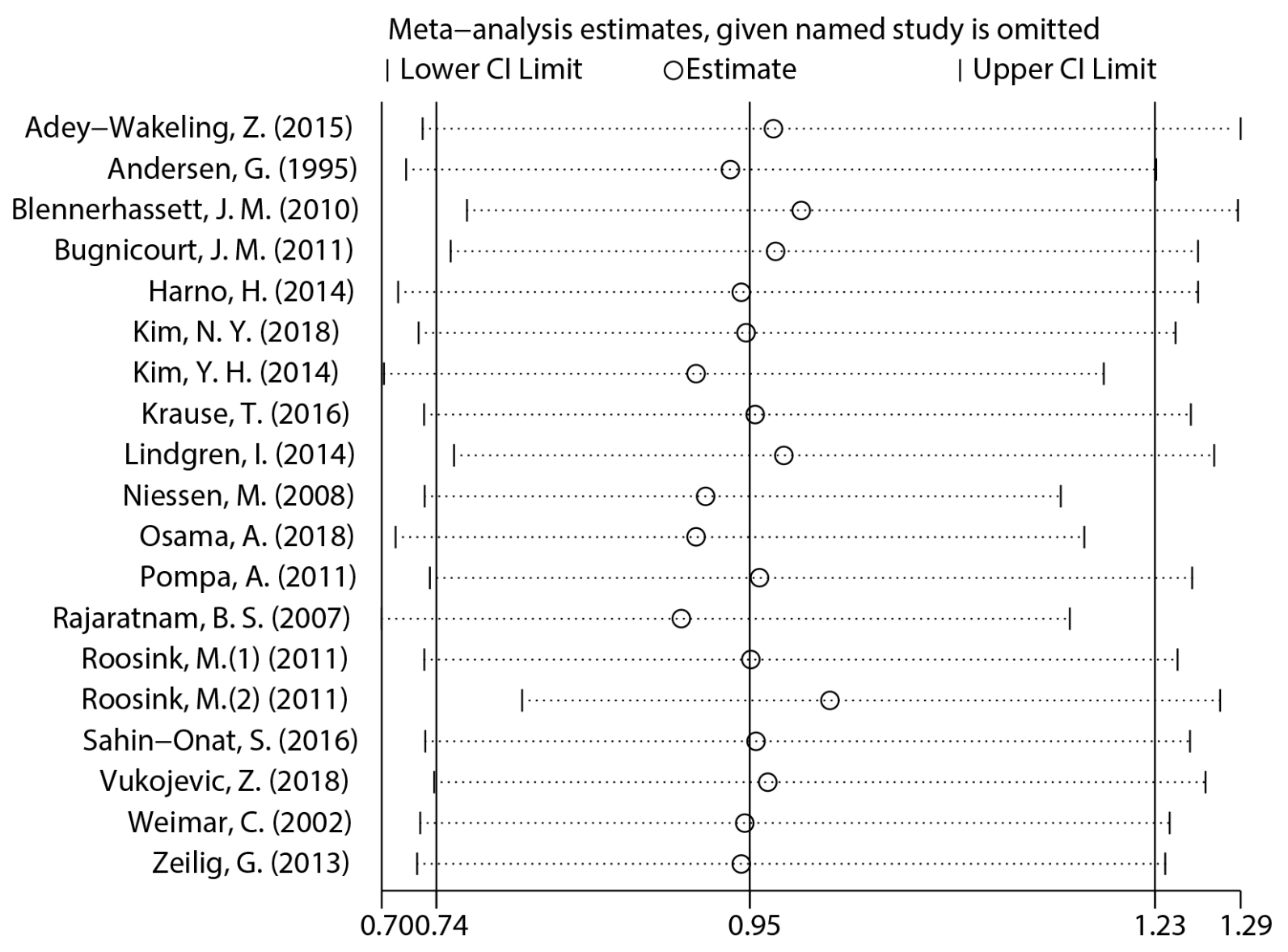

Figure 10

Sensitive analyses of the total study. 


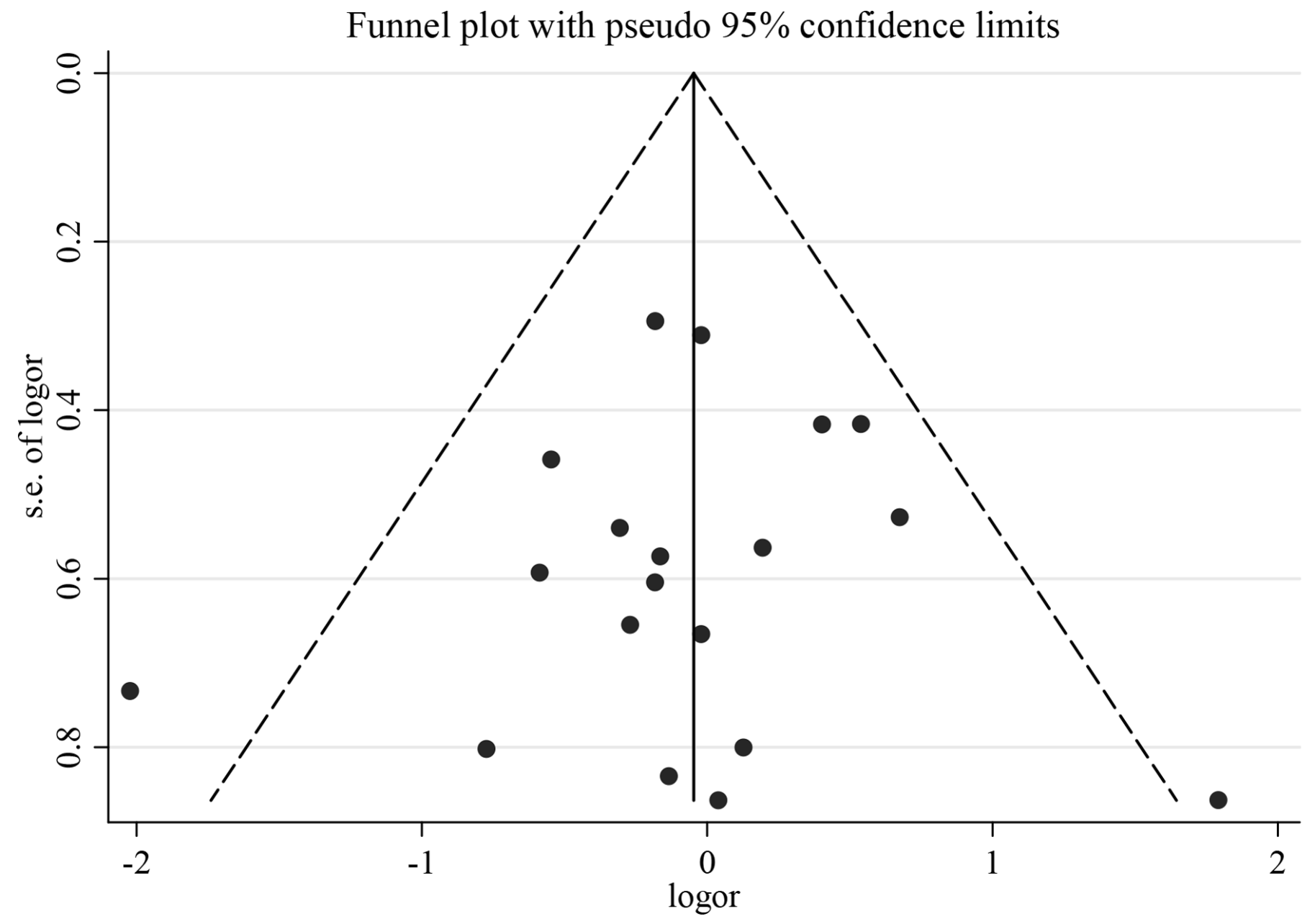

Figure 12

The Funnel blot of the prospective studies. 


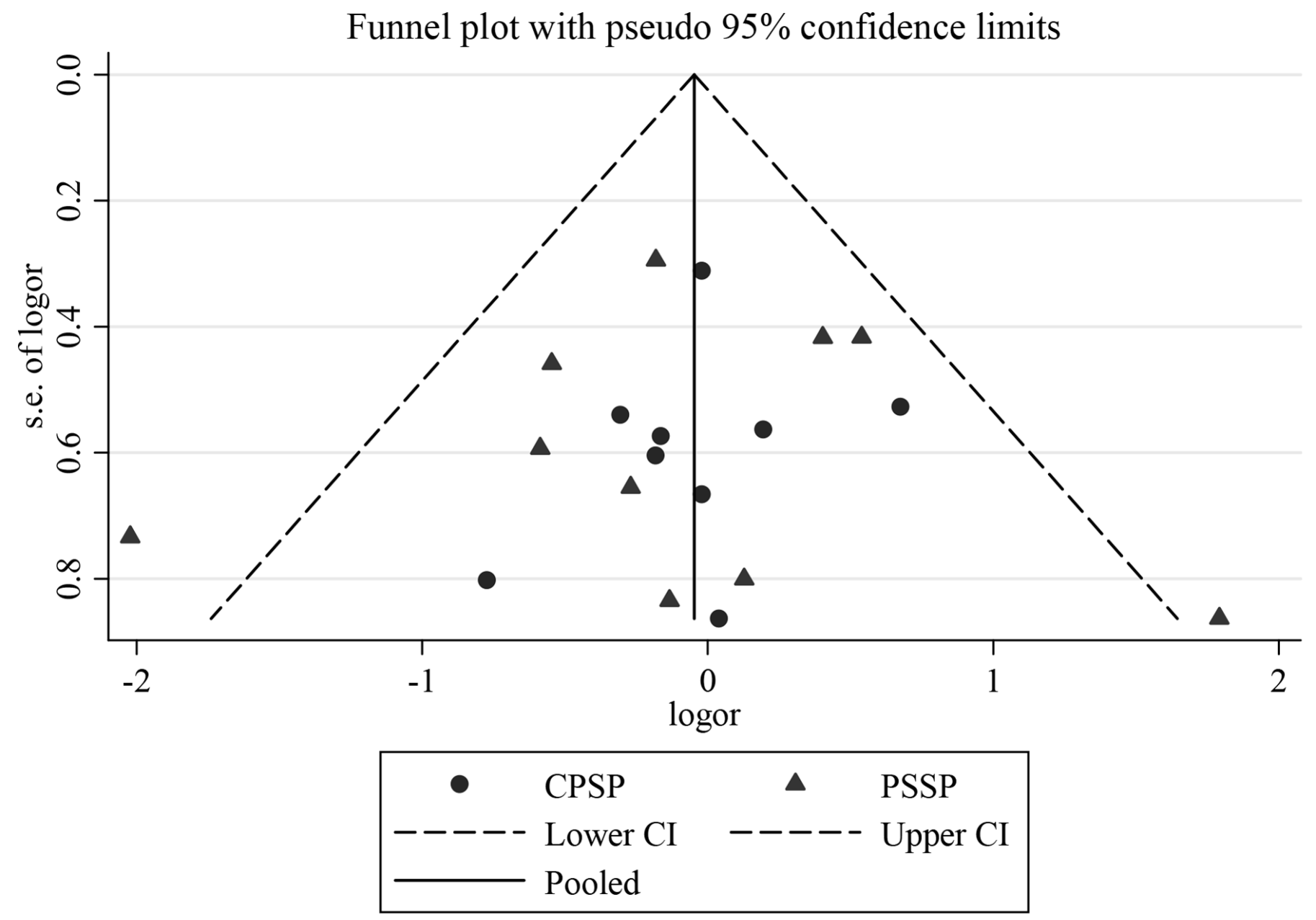

Figure 14

The Funnel blot of the total studies. 


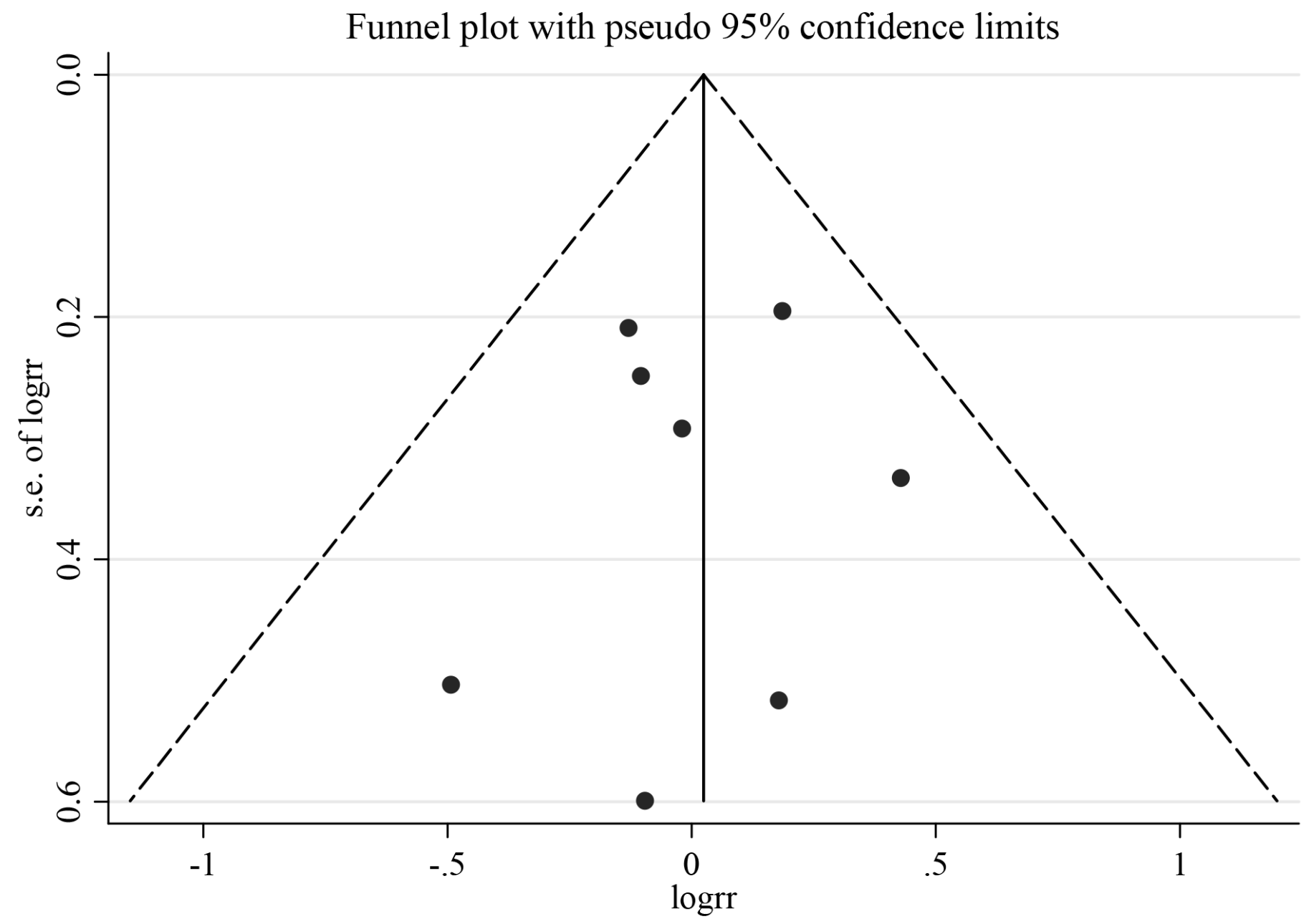

Figure 16

The Funnel blot of the subgroups.

\section{Supplementary Files}

This is a list of supplementary files associated with this preprint. Click to download.

- Additionalfile3TableS3.docx

- Additionalfile1TableS1.docx

- Additionalfile1TableS1.docx

- Additionalfile2TableS2.docx

- Additionalfile3TableS3.docx

- Additionalfile2TableS2.docx 